\title{
A class of functionals possessing multiple global minima
}

\author{
Biagio Ricceri
}

To Professor Gheorghe Moroşanu, with friendship, on his 70th birthday.

Abstract. We get a new multiplicity result for gradient systems. Here is a very particular corollary: Let $\Omega \subset \mathbf{R}^{n}(n \geq 2)$ be a smooth bounded domain and let $\Phi: \mathbf{R}^{2} \rightarrow \mathbf{R}$ be a $C^{1}$ function, with $\Phi(0,0)=0$, such that

$$
\sup _{(u, v) \in \mathbf{R}^{2}} \frac{\left|\Phi_{u}(u, v)\right|+\left|\Phi_{v}(u, v)\right|}{1+|u|^{p}+|v|^{p}}<+\infty
$$

where $p>0$, with $p=\frac{2}{n-2}$ when $n>2$.

Then, for every convex set $S \subseteq L^{\infty}(\Omega) \times L^{\infty}(\Omega)$ dense in $L^{2}(\Omega) \times L^{2}(\Omega)$, there exists $(\alpha, \beta) \in S$ such that the problem

$$
\begin{gathered}
-\Delta u=(\alpha(x) \cos (\Phi(u, v))-\beta(x) \sin (\Phi(u, v))) \Phi_{u}(u, v) \text { in } \Omega \\
-\Delta v=(\alpha(x) \cos (\Phi(u, v))-\beta(x) \sin (\Phi(u, v))) \Phi_{v}(u, v) \text { in } \Omega \\
u=v=0 \text { on } \partial \Omega
\end{gathered}
$$

has at least three weak solutions, two of which are global minima in $H_{0}^{1}(\Omega) \times$ $H_{0}^{1}(\Omega)$ of the functional

$$
\begin{gathered}
(u, v) \rightarrow \frac{1}{2}\left(\int_{\Omega}|\nabla u(x)|^{2} d x+\int_{\Omega}|\nabla v(x)|^{2} d x\right) \\
-\int_{\Omega}(\alpha(x) \sin (\Phi(u(x), v(x)))+\beta(x) \cos (\Phi(u(x), v(x)))) d x .
\end{gathered}
$$

Mathematics Subject Classification (2010): 35J47, 35J50, $49 \mathrm{~K} 35$.

Keywords: Minimax, multiple global minima, variational methods, semilinear elliptic systems. 


\section{Introduction}

Let $S$ be a topological space. A function $g: S \rightarrow \mathbb{R}$ is said to be inf-compact if, for each $r \in \mathbb{R}$, the set $\left.\left.g^{-1}(]-\infty, r\right]\right)$ is compact.

If $Y$ is a real interval and $f: S \times Y \rightarrow \mathbb{R}$ is a function inf-compact and lower semicontinuous in $S$, and concave in $Y$, the occurrence of the strict minimax inequality

$$
\sup _{Y} \inf _{S} f<\inf _{S} \sup _{Y} f
$$

implies that the interior of the set $A$ of all $y \in Y$ for which $f(\cdot, y)$ has at least two local minima is non-empty. This fact was essentially shown in [4], giving then raise to an enormous number of subsequent applications to the multiplicity of solutions for nonlinear equations of variational nature (see [7] for an account up to 2010).

In [6] (see also [5]), we realized that, under the same assumptions as above, the occurrence of the strict minimax inequality also implies the existence of $\tilde{y} \in Y$ such that the function $f(\cdot, \tilde{y})$ has at least two global minima. It may happen that $\tilde{y}$ is unique and does not belong to the closure of $A$ (see Example 7 of [1]).

In [8] and [12], we extended the result of [6] to the case where $Y$ is an arbitrary convex set in a vector space. We also stress that such an extension is not possible for the result of [4]. We then started to build a network of applications of the results of [8] and [12] which touches several different topics: uniquely remotal sets in normed spaces $([8])$; non-expansive operators ([9]); singular points ([10]); Kirchhoff-type problems ([11]); Lagrangian systems of relativistic oscillators ([13]); integral functional of the Calculus of Variations $([14])$; non-cooperative gradient systems $([15])$; variational inequalities $([16])$.

The aim of this paper is to establish a further application within that network.

\section{Results}

The main abstract result is as follows:

Theorem 2.1. Let $X$ be a topological space, $(Y,\langle\cdot, \cdot\rangle$,$) a real Hilbert space, T \subseteq Y$ a convex set dense in $Y$ and $I: X \rightarrow \mathbb{R}, \varphi: X \rightarrow Y$ two functions such that, for each $y \in T$, the function $x \rightarrow I(x)+\langle\varphi(x), y\rangle$ is lower semicontinuous and inf-compact. Moreover, assume that there exists a point $x_{0} \in X$, with $\varphi\left(x_{0}\right) \neq 0$, such that

(a) $x_{0}$ is a global minimum of both functions $I$ and $\|\varphi(\cdot)\|$;

(b) $\inf _{x \in X}\left\langle\varphi(x), \varphi\left(x_{0}\right)\right\rangle<\left\|\varphi\left(x_{0}\right)\right\|^{2}$.

Then, for each convex set $S \subseteq T$ dense in $Y$, there exists $y^{*} \in S$ such that the function $x \rightarrow I(x)+\left\langle\varphi(x), y^{*}\right\rangle$ has at least two global minima in $X$.

Proof. In view of $(b)$, we can find $\tilde{x} \in X$ and $r>0$ such that

$$
I(\tilde{x})+\frac{r}{\left\|\varphi\left(x_{0}\right)\right\|}\left\langle\varphi(\tilde{x}), \varphi\left(x_{0}\right)\right\rangle<I\left(x_{0}\right)+r\left\|\varphi\left(x_{0}\right)\right\| .
$$


Thanks to $(a)$, we have

$$
I\left(x_{0}\right)+r\left\|\varphi\left(x_{0}\right)\right\|=\inf _{x \in X}(I(x)+r\|\varphi(x)\|) .
$$

The function $y \rightarrow \inf _{x \in X}(I(x)+\langle\varphi(x), y\rangle)$ is weakly upper semicontinuous, and so there exists $\tilde{y} \in B_{r}$ such that

$$
\inf _{x \in X}(I(x)+\langle\varphi(x), \tilde{y}\rangle)=\sup _{y \in B_{r}} \inf _{x \in X}(I(x)+\langle\varphi(x), y\rangle),
$$

$B_{r}$ being the closed ball in $X$, centered at 0 , of radius $r$. We distinguish two cases. First, assume that $\tilde{y} \neq \frac{r \varphi\left(x_{0}\right)}{\| \varphi\left(x_{0} \|\right.}$. As a consequence, taking into account that $r\left\|\varphi\left(x_{0}\right)\right\|$ is the maximum of the restriction to $B_{r}$ of the continuous linear functional $\left\langle\varphi\left(x_{0}\right), \cdot\right\rangle$ (attained at the point $\frac{r \varphi\left(x_{0}\right)}{\left\|\varphi\left(x_{0}\right)\right\|}$ only), we have

$$
\inf _{x \in X}(I(x)+\langle\varphi(x), \tilde{y}\rangle) \leq I\left(x_{0}\right)+\left\langle\varphi\left(x_{0}\right), \tilde{y}\right\rangle<I\left(x_{0}\right)+r\left\|\varphi\left(x_{0}\right)\right\| .
$$

Now, assume that $\tilde{y}=\frac{r \varphi\left(x_{0}\right)}{\| \varphi\left(x_{0} \|\right.}$. In this case, due to $(2.1)$, we have

$$
\begin{aligned}
\inf _{x \in X}(I(x)+\langle\varphi(x), \tilde{y}\rangle) \leq & I(\tilde{x})+\langle\varphi(\tilde{x}), \tilde{y}\rangle=I(\tilde{x})+\frac{r}{\left\|\varphi\left(x_{0}\right)\right\|}\left\langle\varphi(\tilde{x}), \varphi\left(x_{0}\right)\right\rangle \\
& <I\left(x_{0}\right)+r\left\|\varphi\left(x_{0}\right)\right\| .
\end{aligned}
$$

Therefore, from (2.2), (2.3), (2.4) and (2.5), it follows that

$$
\sup _{y \in B_{r}} \inf _{x \in X}(I(x)+\langle\varphi(x), y\rangle)<\inf _{x \in X} \sup _{y \in B_{r}}(I(x)+\langle\varphi(x), y\rangle) .
$$

Now, let $S \subseteq T$ be a convex set dense in $Y$. By continuity, we clearly have

$$
\sup _{y \in B_{r} \cap S}\langle\varphi(x), y\rangle=\sup _{y \in B_{r}}\langle\varphi(x), y\rangle
$$

for all $x \in X$. Therefore, in view of (2.6), we have

$$
\begin{aligned}
& \sup _{y \in B_{r} \cap S} \inf _{x \in X}(I(x)+\langle\varphi(x), y\rangle) \leq \sup _{y \in B_{r}} \inf _{x \in X}(I(x)+\langle\varphi(x), y\rangle) \\
< & \inf _{x \in X} \sup _{y \in B_{r}}(I(x)+\langle\varphi(x), y\rangle)=\inf _{x \in X} \sup _{y \in B_{r} \cap S}(I(x)+\langle\varphi(x), y\rangle) .
\end{aligned}
$$

At this point, the conclusion follows directly applying Theorem 1.1 of [12] to the restriction of the function $(x, y) \rightarrow I(x)+\langle\varphi(x), y\rangle$ to $X \times\left(B_{r} \cap S\right)$.

We now present an application of Theorem 2.1 to elliptic systems.

In the sequel, $\Omega \subseteq \mathbf{R}^{n}(n \geq 2)$ is a bounded domain with smooth boundary.

We denote by $\mathcal{A}$ the class of all functions $H: \Omega \times \mathbf{R}^{2} \rightarrow \mathbf{R}$ which are measurable in $\Omega, C^{1}$ in $\mathbf{R}^{2}$ and satisfy

$$
\sup _{(x, u, v) \in \Omega \times \mathbf{R}^{2}} \frac{\left|H_{u}(x, u, v)\right|+\left|H_{v}(x, u, v)\right|}{1+|u|^{p}+|v|^{p}}<+\infty
$$

where $p>0$, with $p<\frac{n+2}{n-2}$ when $n>2$. 
Given $H \in \mathcal{A}$, we are interested in the problem

$$
\begin{aligned}
-\Delta u & =H_{u}(x, u, v) \text { in } \Omega \\
-\Delta v & =H_{v}(x, u, v) \text { in } \Omega \\
u & =v=0 \text { on } \partial \Omega,
\end{aligned}
$$

$H_{u}$ (resp. $H_{v}$ ) denoting the derivative of $H$ with respect to $u$ (resp. $v$ ).

As usual, a weak solution of this problem is any $(u, v) \in H_{0}^{1}(\Omega) \times H_{0}^{1}(\Omega)$ such that

$$
\begin{aligned}
\int_{\Omega} \nabla u(x) \nabla \varphi(x) d x & =\int_{\Omega} H_{u}(x, u(x), v(x)) \varphi(x) d x \\
\int_{\Omega} \nabla v(x) \nabla \psi(x) d x & =\int_{\Omega} H_{v}(x, u(x), v(x)) \psi(x) d x
\end{aligned}
$$

for all $\varphi, \psi \in H_{0}^{1}(\Omega)$.

Define the functional $I_{H}: H_{0}^{1}(\Omega) \times H_{0}^{1}(\Omega) \rightarrow \mathbf{R}$ by

$$
I_{H}(u, v)=\frac{1}{2}\left(\int_{\Omega}|\nabla u(x)|^{2} d x+\int_{\Omega}|\nabla v(x)|^{2} d x\right)-\int_{\Omega} H(x, u(x), v(x)) d x
$$

for all $(u, v) \in H_{0}^{1}(\Omega) \times H_{0}^{1}(\Omega)$.

Since $H \in \mathcal{A}$, the functional $I_{H}$ is $C^{1}$ in $H_{0}^{1}(\Omega) \times H_{0}^{1}(\Omega)$ and its critical points are precisely the weak solutions of the problem. Moreover, due to the Sobolev embedding theorem, the functional $(u, v) \rightarrow \int_{\Omega} H(x, u(x), v(x))$ has a compact derivative and, as a consequence, it is sequentially weakly continuous in $H_{0}^{1}(\Omega) \times H_{0}^{1}(\Omega)$.

Also, we denote by $\lambda_{1}$ the first eigenvalue of the Dirichlet problem

$$
\begin{aligned}
-\Delta u & =\lambda u \text { in } \Omega \\
u & =0 \text { on } \partial \Omega .
\end{aligned}
$$

Our result is as follows:

Theorem 2.2. Let $F, G \in \mathcal{A}$, with $p=\frac{2}{n-2}$ when $n>2$, and let $K \in \mathcal{A}$, with $K(x, 0,0)=0$ for all $x \in \Omega$, satisfy the following conditions: $\left(a_{1}\right)$ one has

$$
\lim _{s^{2}+t^{2} \rightarrow+\infty} \frac{\sup _{x \in \Omega}(|F(x, s, t)|+|G(x, s, t)|)}{s^{2}+t^{2}}=0 ;
$$

$\left(a_{2}\right)$ there is $\left.\eta \in\right] 0, \frac{\lambda_{1}}{2}[$ such that

$$
K(x, s, t) \leq \eta\left(s^{2}+t^{2}\right)
$$

for all $x \in \Omega, s, t \in \mathbf{R}$;

$\left(a_{3}\right)$ one has

$$
\operatorname{meas}\left(\left\{x \in \Omega: 0<|F(x, 0,0)|^{2}+|G(x, 0,0)|^{2}\right\}\right)>0
$$

and

$$
|F(x, 0,0)|^{2}+|G(x, 0,0)|^{2} \leq|F(x, s, t)|^{2}+|G(x, s, t)|^{2}
$$

for all $x \in \Omega, s, t \in \mathbf{R}$; 
$\left(a_{4}\right)$ one has

$$
\begin{aligned}
\operatorname{meas}(\{x \in \Omega & : \inf _{(s, t) \in \mathbf{R}^{2}}(F(x, 0,0) F(x, s, t)+G(x, 0,0) G(x, s, t)) \\
& \left.\left.<|F(x, 0,0)|^{2}+|G(x, 0,0)|^{2}\right\}\right)>0 .
\end{aligned}
$$

Then, for every convex set $S \subseteq L^{\infty}(\Omega) \times L^{\infty}(\Omega)$ dense in $L^{2}(\Omega) \times L^{2}(\Omega)$, there exists $(\alpha, \beta) \in S$ such that the problem

$$
\begin{gathered}
-\Delta u=\alpha(x) F_{u}(x, u, v)+\beta(x) G_{u}(x, u, v)+K_{u}(x, u, v) \text { in } \Omega \\
-\Delta v=\alpha(x) F_{v}(x, u, v)+\beta(x) G_{v}(x, u, v)+K_{v}(x, u, v) \text { in } \Omega \\
u=v=0 \text { on } \partial \Omega
\end{gathered}
$$

has at least three weak solutions, two of which are global minima in $H_{0}^{1}(\Omega) \times H_{0}^{1}(\Omega)$ of the functional

$$
\begin{gathered}
(u, v) \rightarrow \frac{1}{2}\left(\int_{\Omega}|\nabla u(x)|^{2} d x+\int_{\Omega}|\nabla v(x)|^{2} d x\right) \\
-\int_{\Omega}(\alpha(x) F(x, u(x), v(x))+\beta(x) G(x, u(x), v(x))+K(x, u(x), v(x))) d x .
\end{gathered}
$$

Proof. We are going to apply Theorem 2.1, with the following choices: $X$ is the space $H_{0}^{1}(\Omega) \times H_{0}^{1}(\Omega)$ endowed with the weak topology induced by the scalar product

$$
\langle(u, v),(w, \omega)\rangle_{X}=\int_{\Omega}(\nabla u(x) \nabla w(x)+\nabla v(x) \nabla \omega(x)) d x ;
$$

$Y$ is the space $L^{2}(\Omega) \times L^{2}(\Omega)$ with the scalar product

$$
\langle(f, g),(h, k)\rangle_{Y}=\int_{\Omega}(f(x) h(x)+g(x) k(x)) d x ;
$$

$T$ is $L^{\infty}(\Omega) \times L^{\infty}(\Omega) ; I$ is the function defined by

$$
I(u, v)=\frac{1}{2}\left(\int_{\Omega}|\nabla u(x)|^{2} d x+\int_{\Omega}|\nabla v(x)|^{2} d x\right)-\int_{\Omega} K(x, u(x), v(x)) d x
$$

for all $(u, v) \in X ; \varphi$ is the function defined by

$$
\varphi(u, v)=(F(\cdot, u(\cdot), v(\cdot)), G(\cdot, u(\cdot), v(\cdot)))
$$

for all $(u, v) \in X ; x_{0}$ is the zero of $X$. Let us show that the assumptions of Theorem 2.1 are satisfied. First, from (2.7) and (2.8) it clearly follows, respectively, that

$$
\|\varphi(0,0)\|_{Y}^{2}=\int_{\Omega}\left(|F(x, 0,0)|^{2}+\mid G\left(x, 0,\left.0\right|^{2}\right) d x>0\right.
$$

and that

$$
\|\varphi(0,0)\|_{Y}^{2} \leq\|\varphi(u, v)\|_{Y}^{2}
$$

for all $(u, v) \in X$. Moreover, from $\left(a_{2}\right)$, thanks to the Poincaré inequality, we get

$$
\int_{\Omega} K(x, u(x), v(x)) d x \leq \eta \int_{\Omega}\left(|u(x)|^{2}+|v(x)|^{2}\right) d x \leq \frac{\eta}{\lambda_{1}} \int_{\Omega}\left(|\nabla u(x)|^{2}+|\nabla v(x)|^{2}\right) d x
$$


for all $(u, v) \in X$. In particular, since $K(x, 0,0)=0$ in $\Omega$ and $\frac{\eta}{\lambda_{1}}<\frac{1}{2}$, from (2.9) we infer that $(0,0)$ is a global minimum of $I$ in $X$. So, condition $(a)$ is satisfied. Now, let us verify condition $(b)$. To this end, set

$$
P(x, s, t)=F(x, 0,0) F(x, s, t)+G(x, 0,0) G(x, s, t)-|F(x, 0,0)|^{2}-|G(x, 0,0)|^{2}
$$

for all $(x, s, t) \in \Omega \times \mathbf{R}^{2}$ and

$$
D=\left\{x \in \Omega: \inf _{(s, t) \in \mathbf{R}^{2}} P(x, s, t)<0\right\} .
$$

By $\left(a_{4}\right), D$ has a positive measure. In view of the Scorza-Dragoni theorem, there exists a compact set $C \subset D$, with positive measure, such that the restriction of $P$ to $C \times \mathbf{R}^{2}$ is continuous. Fix a point $\tilde{x} \in C$ such that the intersection of $C$ and any ball centered at $\tilde{x}$ has a positive measure. Choose $\tilde{s}, \tilde{t} \in \mathbf{R} \backslash\{0\}$ so that $P(\tilde{x}, \tilde{s}, \tilde{t})<0$. By continuity, there is $r>0$ such that

$$
P(x, \tilde{s}, \tilde{t})<0
$$

for all $x \in C \cap B(\tilde{x}, r)$. Set

$$
\gamma=\sup _{(x, s, t) \in \Omega \times[-|\tilde{s}|,|\tilde{s}|] \times[-|\tilde{t}|,|\tilde{t}|]}|P(x, t, s)| .
$$

Since $F, G \in \mathcal{A}, \gamma$ is finite. Now, choose an open set $A$ such that

$$
C \cap B(\tilde{x}, r) \subset A \subset \Omega
$$

and

$$
\operatorname{meas}(A \backslash(C \cap B(\tilde{x}, r)))<-\frac{\int_{C \cap B(\tilde{x}, r)} P(x, \tilde{s}, \tilde{t}) d x}{\gamma} .
$$

Finally, choose two functions $\tilde{u}, \tilde{v} \in H_{0}^{1}(\Omega)$ such that

$$
\tilde{u}(x)=\tilde{s}, \quad \tilde{v}(x)=\tilde{t}
$$

for all $x \in C \cap B(\tilde{x}, r)$,

for all $x \in \Omega \backslash A$ and

$$
\tilde{u}(x)=\tilde{v}(x)=0
$$

$$
|\tilde{u}(x)| \leq|\tilde{s}|,|\tilde{v}(x)| \leq|\tilde{t}|
$$

for all $x \in \Omega$. Then, taking (2.10) into account, we have

$$
\begin{aligned}
& \langle\varphi(\tilde{u}, \tilde{v}), \varphi(0,0)\rangle_{Y}-\|\varphi(0,0)\|_{Y}^{2}=\int_{\Omega} P(x, \tilde{u}(x), \tilde{v}(x)) d x \\
= & \int_{C \cap B(\tilde{x}, r)} P(x, \tilde{s}, \tilde{t}) d x+\int_{A \backslash(C \cap B(\tilde{x}, r))} P(x, \tilde{u}(x), \tilde{v}(x)) d x \\
< & \int_{C \cap B(\tilde{x}, r)} P(x, \tilde{s}, \tilde{t}) d x+\gamma \operatorname{meas}(A \backslash(C \cap B(\tilde{x}, r))<0 .
\end{aligned}
$$

This shows that $(b)$ is satisfied. Finally, fix $\alpha, \beta \in L^{\infty}(\Omega)$. Clearly, the function

$$
(x, s, t) \rightarrow \alpha(x) F(x, s, t)+\beta(x) F(x, s, t)+K(x, s, t)
$$


belongs to $\mathcal{A}$, and so the functional

$$
(u, v) \rightarrow I(u, v)+\langle\varphi(u, v),(\alpha, \beta)\rangle_{Y}
$$

is sequentially weakly lower semicontinuous in $X$. Let us show that it is coercive. Set

$$
\theta=\max \left\{\|\alpha\|_{L^{\infty}(\Omega)},\|\beta\|_{L^{\infty}(\Omega)}\right\}
$$

and fix $\epsilon>0$ so that

$$
\epsilon<\frac{1}{\theta}\left(\frac{\lambda_{1}}{2}-\eta\right) .
$$

By $\left(a_{1}\right)$, there is $c_{\epsilon}>0$ such that

$$
|F(x, s, t)|+|G(x, s, t)| \leq \epsilon\left(|s|^{2}+|t|^{2}\right)+c_{\epsilon}
$$

for all $(x, s, t) \in \Omega \times \mathbf{R}^{2}$. Then, for each $u, v \in H_{0}^{1}(\Omega)$, recalling (2.9), we have

$$
\begin{gathered}
I(u, v)+\langle\varphi(u, v),(\alpha, \beta)\rangle_{Y} \\
\geq\left(\frac{1}{2}-\frac{\eta}{\lambda_{1}}\right) \int_{\Omega}\left(|\nabla u(x)|^{2}+|\nabla v(x)|^{2}\right) d x \\
-\int_{\Omega}|\alpha(x) F(x, u(x), v(x))+\beta(x) G(x, u(x), v(x))| d x \\
\geq\left(\frac{1}{2}-\frac{\eta}{\lambda_{1}}\right) \int_{\Omega}\left(|\nabla u(x)|^{2}+|\nabla v(x)|^{2}\right) d x-\theta \epsilon \int_{\Omega}\left(|u(x)|^{2}+|v(x)|^{2}\right) d x-\theta c_{\epsilon} \operatorname{meas}(\Omega) \\
\geq\left(\frac{1}{2}-\frac{\eta}{\lambda_{1}}-\frac{\theta \epsilon}{\lambda_{1}}\right) \int_{\Omega}\left(|\nabla u(x)|^{2}+|\nabla v(x)|^{2}\right) d x-\theta c_{\epsilon} \operatorname{meas}(\Omega) .
\end{gathered}
$$

Notice that, in view of $(2.11)$, we have $\frac{1}{2}-\frac{\eta}{\lambda_{1}}-\frac{\theta \epsilon}{\lambda_{1}}>0$, and so

$$
\lim _{\|(u, v)\|_{X} \rightarrow+\infty}\left(I(u, v)+\langle\varphi(u, v),(\alpha, \beta)\rangle_{Y}\right)=+\infty,
$$

as claimed.

In particular, this also implies that the functional $(u, v) \rightarrow I(u, v)+\langle\varphi(u, v),(\alpha, \beta)\rangle_{Y}$ is weakly lower semicontinuous, by the Eberlein-Smulyan theorem. Thus, the assumptions of Theorem 2.1 are satisfied. Therefore, for each convex set $S \subseteq L^{\infty}(\Omega) \times L^{\infty}(\Omega)$ dense in $H_{0}^{1}(\Omega) \times H_{0}^{1}(\Omega)$, there exists $(\alpha, \beta) \in S$, such that the functional

$$
\begin{gathered}
(u, v) \rightarrow \frac{1}{2}\left(\int_{\Omega}|\nabla u(x)|^{2} d x+\int_{\Omega}|\nabla v(x)|^{2} d x\right) \\
-\int_{\Omega}(\alpha(x) F(x, u(x), v(x))+\beta(x) G(x, u(x), v(x))+K(x, u(x), v(x))) d x
\end{gathered}
$$

has at least two global minima in $H_{0}^{1}(\Omega) \times H_{0}^{1}(\Omega)$. Finally, by Example 38.25 of [17], the same functional satisfies the Palais-Smale condition, and so it admits at least three critical points, in view of Corollary 1 of [3]. The proof is complete.

Remark 2.3. We are not aware of known results close enough to Theorem 2.2 in order to do a proper comparison. This sentence also applies to the case of single equations, that is to say when $F, G, K$ depend on $x$ and $s$ only. For an account on elliptic systems, we refer to $[2]$. 
Among the various corollaries of Theorem 2.2, we wish to stress the following ones:

Corollary 2.4. Let $K \in \mathcal{A}$, with $K(x, 0,0)=0$ for all $x \in \Omega$, satisfy condition $\left(a_{2}\right)$. Moreover, let $\Phi: \mathbf{R}^{2} \rightarrow \mathbf{R}$ be a non-constant $C^{1}$ function, with $\Phi(0,0)=0$, belonging to $\mathcal{A}$, with $p=\frac{2}{n-2}$ when $n>2$.

Then, for every convex set $S \subseteq L^{\infty}(\Omega) \times L^{\infty}(\Omega)$ dense in $L^{2}(\Omega) \times L^{2}(\Omega)$, there exists $(\alpha, \beta) \in S$ such that the problem

$$
\begin{gathered}
-\Delta u=(\alpha(x) \cos (\Phi(u, v))-\beta(x) \sin (\Phi(u, v))) \Phi_{u}(u, v)+K_{u}(x, u, v) \text { in } \Omega \\
-\Delta v=(\alpha(x) \cos (\Phi(u, v))-\beta(x) \sin (\Phi(u, v))) \Phi_{v}(u, v)+K_{v}(x, u, v) \text { in } \Omega \\
u=v=0 \text { on } \partial \Omega
\end{gathered}
$$

has at least three weak solutions, two of which are global minima in $H_{0}^{1}(\Omega) \times H_{0}^{1}(\Omega)$ of the functional

$$
\begin{gathered}
(u, v) \rightarrow \frac{1}{2}\left(\int_{\Omega}|\nabla u(x)|^{2} d x+\int_{\Omega}|\nabla v(x)|^{2} d x\right) \\
-\int_{\Omega}(\alpha(x) \sin (\Phi(u(x), v(x)))+\beta(x) \cos (\Phi(u(x), v(x)))+K(x, u(x), v(x))) d x .
\end{gathered}
$$

Proof. It suffices to apply Theorem 2.2 to the functions $F, G: \mathbf{R}^{2} \rightarrow \mathbf{R}$ defined by

$$
\begin{aligned}
& F(s, t)=\sin (\Phi(s, t)), \\
& G(s, t)=\cos (\Phi(s, t))
\end{aligned}
$$

for all $(s, t) \in \mathbf{R}^{2}$.

Corollary 2.5. Let $F, G: \mathbf{R} \rightarrow \mathbf{R}$ belong to $\mathcal{A}$, with $p=\frac{2}{n-2}$ when $n>2$. Moreover, assume that $F, G$ are twice differentiable at 0 and that

$$
\begin{gathered}
\lim _{|s| \rightarrow+\infty} \frac{|F(s)|+|G(s)|}{s^{2}}=0, \\
0<|F(0)|^{2}+|G(0)|^{2}=\inf _{s \in \mathbf{R}}\left(|F(s)|^{2}+|G(s)|^{2}\right), \\
F^{\prime \prime}(0) F(0)+G^{\prime \prime}(0) G(0)<0 .
\end{gathered}
$$

Then, for every convex set $S \subseteq L^{\infty}(\Omega) \times L^{\infty}(\Omega)$ dense in $L^{2}(\Omega) \times L^{2}(\Omega)$, there exists $(\alpha, \beta) \in S$ such that the problem

$$
\begin{gathered}
-\Delta u=\alpha(x) F^{\prime}(u)+\beta(x) G^{\prime}(u) \text { in } \Omega \\
u=0 \text { on } \partial \Omega
\end{gathered}
$$

has at least three weak solutions, two of which are global minima in $H_{0}^{1}(\Omega)$ of the functional

$$
u \rightarrow \frac{1}{2} \int_{\Omega}|\nabla u(x)|^{2} d x-\int_{\Omega}(\alpha(x) F(u(x))+\beta(x) G(u(x))) d x .
$$


Proof. We apply Theorem 2.2 taking $K=0$. Since 0 is a global minimum of the function $|F(\cdot)|^{2}+|G(\cdot)|^{2}$, we have

$$
F^{\prime}(0) F(0)+G^{\prime}(0) G(0)=0
$$

and so, in view of (2.12), 0 is a strict local maximum for the function

$$
F(\cdot) F(0)+G(\cdot) G(0) .
$$

Hence, $\left(a_{4}\right)$ is satisfied and Theorem 2.2 gives the conclusion.

\section{References}

[1] Cabada, A., Iannizzotto, A., A note on a question of Ricceri, Appl. Math. Lett., 25(2012), 215-219.

[2] de Figueiredo, D.G., Semilinear elliptic systems: existence, multiplicity, symmetry of solutions, Handbook of Differential Equations: Stationary Partial Differential Equations, Vol. V, 1-48, Handb. Differ. Equ., Elsevier/North-Holland, 2008.

[3] Pucci, P., Serrin, J., A mountain pass theorem, J. Differential Equations, 60(1985), 142149.

[4] Ricceri, B., On a three critical points theorem, Arch. Math., 75(2000), 220-226.

[5] Ricceri, B., Well-posedness of constrained minimization problems via saddle-points, J. Global Optim., 40(2008), 389-397.

[6] Ricceri, B., Multiplicity of global minima for parametrized functions, Rend. Lincei Mat. Appl., 21(2010), 47-57.

[7] Ricceri, B., Nonlinear eigenvalue problems, in "Handbook of Nonconvex Analysis and Applications" D. Y. Gao and D. Motreanu eds., 543-595, International Press, 2010.

[8] Ricceri, B., A strict minimax inequality criterion and some of its consequences, Positivity, 16(2012), 455-470.

[9] Ricceri, B., A range property related to non-expansive operators, Mathematika, 60(2014), $232-236$.

[10] Ricceri, B., Singular points of non-monotone potential operators, J. Nonlinear Convex Anal., 16(2015), 1123-1129.

[11] Ricceri, B., Energy functionals of Kirchhoff-type problems having multiple global minima, Nonlinear Anal., 15(2015), 130-136.

[12] Ricceri, B., On a minimax theorem: an improvement, a new proof and an overview of its applications, Minimax Theory Appl., 2(2017), 99-152.

[13] Ricceri, B., Another multiplicity result for the periodic solutions of certain systems, Linear Nonlinear Anal., 5(2019), 371-378.

[14] Ricceri, B., Miscellaneous applications of certain minimax theorems II, Acta Math. Vietnam., 45(2020), 515-524.

[15] Ricceri, B., An alternative theorem for gradient systems, Pure Appl. Funct. Anal. (to appear).

[16] Ricceri, B., A remark on variational inequalities in small balls, J. Nonlinear Var. Anal., 4(2020), 21-26.

[17] Zeidler, E., Nonlinear functional analysis and its applications, vol. III, Springer-Verlag, 1985. 


\section{Biagio Ricceri}

Department of Mathematics and Informatics,

University of Catania,

Viale A. Doria 6,

95125 Catania, Italy

e-mail: ricceri@dmi.unict.it 